\title{
OPTIMIZACIÓN HEURÍSTICA CON CRITERIOS DE ERROR DE CONTROL TMD EN TURBINAS MARINAS FLOTANTES
}

\author{
Juan Almenara Ahijón \\ Universidad Complutense de Madrid, España \\ juanalme@ucm.es \\ Matilde Santos Peñas \\ Universidad Complutense de Madrid, España \\ msantos@ucm.es \\ María Tomás-Rodríguez \\ City, University of London, UK \\ Maria.Tomas-Rodriguez.1@city.ac.uk
}

\begin{abstract}
Resumen
De entre el despliegue actual de energías renovables, las turbinas eólicas flotantes son un recurso prometedor. Permiten sacar el máximo rendimiento al viento que se produce en alta mar, donde alcanza una mayor y más constante velocidad. Sin embargo, al estar situadas en aguas profundas están sometidas a fuertes cargas, causadas principalmente por las olas y el mismo viento. Para reducir las vibraciones que se producen en la estructura se pueden usar dispositivos de control estructural, que provienen de la ingeniería civil. Pero su diseño y sintonía no son tareas sencillas debido a los diversos objetivos que coexisten en la aplicación de estos sistemas de control pasivo. En este trabajo se explora el uso de distintos métodos de análisis de señales para la optimización de dispositivos TMD (Tuned Mass Damper) mediante algoritmos genéticos. Se han aplicado diversos criterios de error para la optimización con el fin de obtener una mayor reducción de vibraciones en los diversos elementos de la turbina flotante.
\end{abstract}

Palabras clave: Modelado y Simulación, Optimización, Turbinas eólicas marinas flotantes, Algoritmos genéticos, Energía renovable.

\section{INTRODUCCIÓN}

La energía eólica marina generada por turbinas flotantes es una fuente de energía limpia y renovable, ubicada mar adentro, donde se reduce el impacto visual $\mathrm{y}$ acústico en comparación con los aerogeneradores en tierra o incluso con las turbinas offshore que están ancladas al suelo marino, cercanas a la costa. Además cuentan con superficies extensas donde desplegar los parques eólicos y con facilidad de transporte al no tener limitaciones respecto a la carga o las dimensiones. Su principal desventaja es el coste económico de este tipo de proyectos y que, al ser un tema todavía abierto a la investigación, hay pocos datos experimentales para validar los trabajos que se realizan sobre ellas [9].

Las turbinas eólicas marinas flotantes (FOWT, de sus siglas en inglés, Floating Offshore Wind Turbine) se pueden categorizar en función de su estructura en tres grandes grupos, según su método de estabilización (Fig.1): torres flotantes sobre plataformas, turbinas flotantes estabilizadas mediante cables anclados en tensión al lecho marino, y las estabilizadas mediante un balastro.

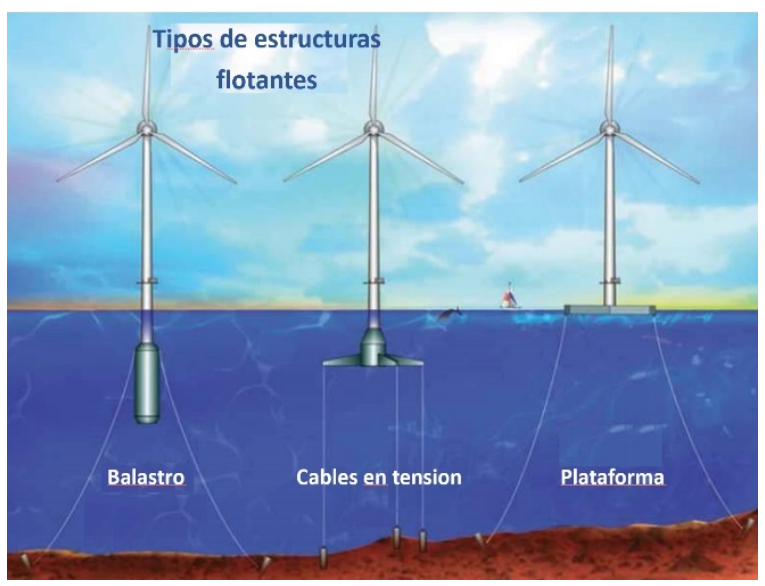

Figura 1: Diseños de turbinas flotantes.

Debido a las condiciones extremas a las que se pueden ver sometidas estas turbinas en altamar, sobre todo de fuertes vientos, oleajes y corrientes marinas, están sometidas a cargas intensas y vibraciones que pueden resultar significativas, no sólo para la propia estructura de la turbina sino para la generación de electricidad. 
En este trabajo se plantea, a partir de un modelo de turbina flotante con plataforma, donde se ha implementado un sistema de control estructural TMD (Tuned Mass Dumper), llevar a cabo la optimización de los parámetros del dispositivo de control aplicando diversos criterios de error para las señales del sistema.

Para resolver este problema de optimización se han utilizado Algoritmos Genéticos (AG) ya que permiten calcular los parámetros del TMD sin conocer la frecuencia estructural del sistema.

La estructura del artículo es la siguiente. Tras esta introducción, en la sección 2 se presenta el modelo matemático de la turbina flotante y del dispositivo de control pasivo TMD utilizados. En la sección 3 se describe la configuración del AG aplicado y los criterios de error usados para la optimización. En la sección 4 se analizan y comentan los resultados obtenidos de la simulación del sistema con el control. El trabajo termina con las conclusiones y trabajos futuros.

\section{MODELO MATEMÁTICO DE LA TURBINA Y EL TMD}

Para la simulación y la optimización se ha usado el modelo no lineal de una turbina flotante con plataforma, donde se consideran despreciables las fuerzas hidrodinámicas y aerodinámicas, ya que el objetivo es estudiar la reducción de fatiga causada en la torre. Este modelo de la turbina flotante [3] está ampliamente aceptado como referencia para la investigación del comportamiento y movimiento de las FOWTs. Recientemente ha sido utilizado por los autores para desarrollar trabajos de investigación dentro del ámbito presentado en esta contribución [10, $11]$.

La plataforma y la torre, elementos que forman la turbina, tienen cada una un grado de libertad angular, mientras que el TMD tiene un grado de libertad de traslación. El modelado incorpora las perturbaciones en la dinámica de la plataforma, la torre y el dispositivo TMD, pero no incluye las palas de la turbina ni las líneas de amarre ya que en estudios previos se han obtenido resultados que indican que no resulta un factor influyente en la vibración de la estructura [8].

\subsection{MODELO MATEMÁTICO TURBINA}

Para estudiar la dinámica estructural del sistema se utiliza un modelo formado por tres masas que representan la torre, la plataforma y el dispositivo TMD.
La plataforma está conectada a la torre mediante un resorte giratorio y un amortiguador, que representan la fuerza de restauración hidrostática y la amortiguación del agua, respectivamente. La torre también se modela como un resorte giratorio y un amortiguador que representan la torsión y flexibilidad estructural, mientras que el TMD presenta un movimiento lineal sobre un único eje [7].

En las ecuaciones diferenciales que modelan este sistema, (1-3), los subíndices $t$ hacen referencia a la torre, $b$ a la plataforma (barge) y TMD al dispositivo de control estructural. Los términos correspondientes a fuerzas hidrostáticas de restauración y amortiguación del agua se puede modelar como un resorte giratorio $\left(k_{b}\right)$ y un amortiguador $\left(d_{b}\right)$, unidos a la plataforma [12]. Se han linealizado las funciones trigonométricas ya que los ángulos máximos de desplazamiento que se han alcanzado en estas estructuras en condiciones normales no superan los $10^{\circ}$. Se obtienen las siguientes ecuaciones:

$$
\begin{gathered}
I_{b} \ddot{\theta}_{b}=-d_{b} \dot{\theta_{b}}-k_{b} \theta_{b}-m_{b} g R_{b} \theta_{b}+k_{t}\left(\theta_{t}-\theta_{b}\right)+ \\
d_{t}\left(\dot{\theta}_{t}-\dot{\theta}_{b}\right) \\
I_{t} \ddot{\theta}_{t}=m_{t} g R_{t} \theta_{t}-k_{t}\left(\theta_{t}-\theta_{b}\right)-d_{t}\left(\dot{\theta_{t}}-\theta_{b}\right)- \\
k_{T M D} R_{T M D}\left(R_{T M D} \theta_{t}-x_{T M D}\right)- \\
d_{T M D} R_{T M D}\left(R_{T M D} \dot{\theta}_{t}-x_{T M D}\right)-m_{T M D} g\left(R_{T M D} \theta_{t}-\right. \\
\left.x_{T M D}\right) \\
m_{T M D} x_{T M D}=k_{T M D}\left(R_{T M D} \theta_{t}-x_{T M D}\right)+ \\
d_{T M D}\left(R_{T M D} \dot{\theta}_{t}-x_{T M D}\right)+d_{T M D}
\end{gathered}
$$

En la Tabla 1 se describen las principales variables y sus unidades.

Tabla 1: Parámetros del sistema para el modelo de la FOWT con TMD.

\begin{tabular}{|c|c|}
\hline Símbolo & Variable \\
\hline$I_{t, b}$ & Inercia $\left[\mathrm{kgm}^{2}\right]$ \\
\hline$k_{t, b}$ & Rigidez $\left[\mathrm{kgm}^{2} / \mathrm{s}\right]$ \\
\hline$d_{t, b}$ & Amortiguamiento $\left[\mathrm{kgm}^{2} / \mathrm{s}^{2}\right]$ \\
\hline$R_{t, b, T M D}$ & $\begin{array}{r}\text { Distancia eje de masa- eje de } \\
\text { rotación }[\mathrm{m}]\end{array}$ \\
\hline$\theta_{t}$ & Angulo desplazamiento torre $\left[^{\circ}\right]$ \\
\hline$\theta_{b}$ & Angulo desplazamiento plataforma $\left[^{\circ}\right]$ \\
\hline$x_{T M D}$ & Desplazamiento TMD $\left[\mathrm{m}^{\circ}\right]$ \\
\hline$d_{T M D}$ & Amortiguamiento TMD $\left[\mathrm{kgm}^{2} / \mathrm{s}^{2}\right]$ \\
\hline$k_{T M D}$ & Rigidez TMD $\left[\mathrm{kgm}{ }^{2} / \mathrm{s}\right]$ \\
\hline$m_{T M D, t, b}$ & Masa $[\mathrm{kg}]$ \\
\hline
\end{tabular}

\subsection{CONTROL ESTRUCTURAL}

En este trabajo se aplica un sistema de control pasivo, el dispositivo TMD. Es uno de los métodos más utilizados e implementados en ingeniería civil ya que 
es muy eficiente a la hora de reducir vibraciones. Está formado por un muelle, un amortiguador y una masa (Fig. 2). El dispositivo se desplaza longitudinalmente sobre la estructura, a una frecuencia de sintonía que coincide con la frecuencia de resonancia de la turbina flotante. En las FOWT se suele instalar en la góndola.

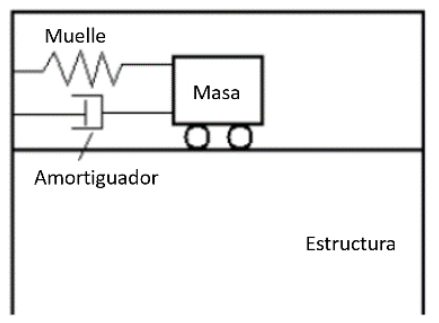

Figura 2: Estructura del TMD

De esta forma, se logra disipar la energía producida por la oscilación de la estructura (Fig. 3).
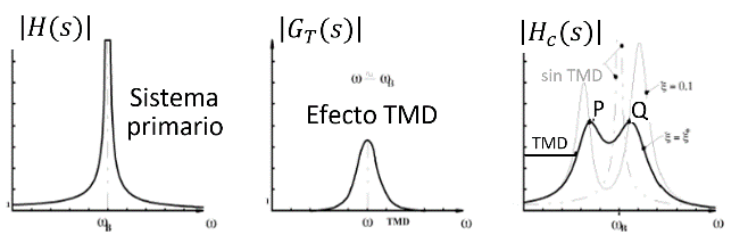

Figura 3: Efecto del TMD

\section{OPTIMIZACIÓN MEDIANTE AG}

DEL TMD

Uno de los métodos heurísticos más utilizados para la optimización son los Algoritmos Genéticos (AG) [1]. En este problema se va a aplicar esta técnica para encontrar los valores óptimos del sistema TMD, de forma que se reduzca lo más posible la oscilación de la estructura.

Se podría también utilizar la frecuencia de resonancia para amortiguarla, pero eso requiere saber de antemano la frecuencia de la estructura, limitando la posible generalización del método ya que es necesario conocerla para cada FOWT concreta. Otros algoritmos de búsqueda convencionales no se han aplicado porque son computacionalmente muy exigentes, y utilizarlos para optimizar este problema sería una tarea larga y pesada. Por eso se ha decido utilizar una técnica evolutiva, como son los algoritmos genéticos, que permiten definir de forma bastante flexible la función objetivo a optimizar con los parámetros buscados [6].

El algoritmo genético es un método para resolver problemas de optimización basándose en la selección natural. El algoritmo modifica repetidamente una población de soluciones (individuos). En cada generación, el algoritmo selecciona a algunos individuos de la población actual para ser padres y los utiliza para producir la siguiente generación (mediante el cruce), junto con otros operadores genéticos como mutación y/o reemplazamiento. Durante iteraciones sucesivas la generación evoluciona hacia la solución óptima [5].

\subsection{FUNCIÓN DE COSTE}

Los tres parámetros a optimizar en este caso son la masa, la constante de amortiguamiento y la rigidez del dispositivo TMD.

Para reducir la oscilación sobre la plataforma se utiliza como función a optimizar el ángulo de desplazamiento de la torre con respecto a la plataforma, concretamente se define éste como señal de error. Es decir, se usa $\theta_{t}$ como señal a minimizar, que se puede definir como el desplazamiento longitudinal del extremo de la torre, tomando como origen el eje perpendicular de la plataforma.

Se va a aplicar como función de coste la definición de cuatro criterios de error que se utilizan con frecuencia para analizar el funcionamiento de los sistemas de control [4]. Se estudia el error en el estado estacionario, puesto que se pretende reducir la amplitud y frecuencia de las oscilaciones.

Los criterios de error que se han usado como función de optimización del algoritmo genético son:

Integral de error cuadrático

$$
\operatorname{ISE}=\int_{0}^{\infty} \mathrm{e}^{2}(\mathrm{t}) \mathrm{dt}
$$

Integral de error absoluto

$$
\mathrm{IAE}=\int_{0}^{\infty}|\mathrm{e}(\mathrm{t})| \mathrm{dt}
$$

Error cuadrático integral por el tiempo

$$
\operatorname{ITSE}=\int_{0}^{\infty} \mathrm{t} \cdot \mathrm{e}^{2}(\mathrm{t}) \mathrm{dt}
$$

Producto del error absoluto por el tiempo

$$
\operatorname{ITAE}=\int_{0}^{\infty} \mathrm{t}|\mathrm{e}(\mathrm{t})| \mathrm{dt}
$$

La configuración del AG es la siguiente (Tabla 2):

Tabla 2: Configuración del AG.

\begin{tabular}{|l|l|}
\hline No. Individuos población & 50 \\
\hline No. Generaciones & 15 \\
\hline Elitisimo (\%) & $5 \%$ \\
\hline Probabilidad de cruce & 0.8 \\
\hline
\end{tabular}


En este caso, no se ha implementado el operador mutación ya que utilizar una función híbrida excluiría las restricciones declaradas en la función de coste.

\section{SIMULACIÓN Y RESULTADOS}

Para realizar la simulación se ha usado el software Matlab, y para la optimización la ToolBox de Algoritmos Genéticos, con los valores de la Tabla 2.

Se ha implementado el modelo matemático descrito en las ecuaciones (1-3) con los valores de simulación recogidos en la Tabla 3 para el modelo de turbina desarrollado por NREL de 5-MW, equipada con tres palas sobre una plataforma flotante [3].

Tabla 3: Parámetros de la FOWT.

\begin{tabular}{|c|c|c|c|}
\hline \multicolumn{2}{|c|}{ Base } & \multicolumn{2}{|c|}{ Torre } \\
\hline$I_{b}\left(\mathrm{kgm}^{2}\right)$ & $1.76667 \times 10^{9}$ & $I_{t}\left(\mathrm{kgm}^{2}\right)$ & $3.3428 \times 10^{9}$ \\
\hline$m_{b}(\mathrm{~kg})$ & 5452000 & $m_{t}(\mathrm{~kg})$ & 6797460 \\
\hline$k_{b}\left(\mathrm{kgm}^{2} / \mathrm{s}^{2}\right)$ & $1.888 \times 10^{9}$ & $k_{f}\left(\mathrm{kgm}^{2} / \mathrm{s}^{2}\right)$ & $1.2519 \times 10$ \\
\hline$d_{b}\left(\mathrm{kgm}^{2} / \mathrm{s}\right)$ & $5.123 \times 10^{7}$ & $d_{t}\left(\mathrm{kgm}^{2} / \mathrm{s}\right)$ & $2.869 \times 10^{7}$ \\
\hline$R_{b}(\mathrm{~m})$ & 0.281 & $R_{t}(\mathrm{~m})$ & 64.2 \\
\hline & \multicolumn{2}{|l|}{ TMD } & \\
\hline & $k_{T M D}\left(\mathrm{kgm}^{2} / \mathrm{s}^{2}\right)$ & 28805 & \\
\hline & $m_{T M D}(\mathrm{~kg})$ & 40000 & \\
\hline & $d_{T M D}\left(\mathrm{kgm}^{2} / \mathrm{s}\right)$ & 10183 & \\
\hline & $R_{T M D}(\mathrm{~m})$ & 90.6 & \\
\hline
\end{tabular}

Para el TMD partimos de los parámetros recogidos en el trabajo de [8], representados en la Tabla 3, con los que se comparará la respuesta obtenida.

Para acotar el proceso de optimización se han aplicado restricciones al espacio de solución de cada una de las variables del TMD considerando unos intervalos realistas para la implementación física de estos elementos.

$$
\begin{gathered}
k_{t m d} \in[15000-25000] \mathrm{kgm}^{2} / \mathrm{s}^{2} \\
d_{t m d} \in[5000-15000] \mathrm{kgm}^{2} / \mathrm{s} \\
m_{t m d} \in[15000-40000] \mathrm{kg}
\end{gathered}
$$

\subsection{RESULTADOS DE SIMULACIÓN CON LOS CRITERIOS DE ERROR}

En la Fig. 4 se muestra el resultado del proceso de optimización del TMD mediante GA utilizando como función objetivo, a modo de ejemplo, el error ISE. En la gráfica de arriba se representa el mejor valor obtenido para la función de coste, y la media en cada iteración. Se puede observar que convergen rápidamente; prácticamente a partir de la $8^{\mathrm{a}}$ generación la solución es siempre la misma. No se encuentra una solución que pueda disminuir la función de coste basada en el error de la señal.

La segunda grafica muestra la diferencia media entre los resultados obtenidos en cada población. Se aprecia que según se va acercando la media al valor óptimo, la distancia entre los valores de los individuos de la población se reduce, es decir, cada vez las soluciones son más homogéneas. El último gráfico representa el histograma según el cual se distribuyen los valores obtenidos en la última población generada. En general se puede ver que es un método rápido y que requiere poco tiempo de computación.
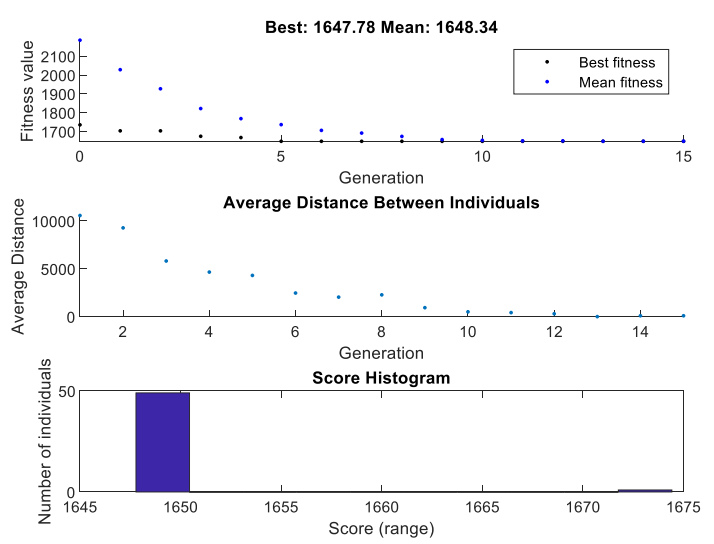

Figura 4: Comportamiento del AG para la función criterio de error ISE.

Para los otros criterios de error, IAE, ITSE e ITAE, los resultados son similares. Con el criterio del error ITAE el número de generaciones que tarda en converger es ligeramente menor, en torno a 7; con el IAE es también 7-8, y con ITSE está alrededor de 5. Respecto al histograma, en general presentan poca dispersión y la solución está concentrada en uno o dos valores (ITAE, ITSE) excepto con IAE, aunque en todos los casos el rango de valores de la solución es muy pequeño.

Aplicando la optimización al error cuadrático ISE se obtienen los resultados presentados en la Tabla 5 para los parámetros del TMD.

Tabla 5: Valores óptimos obtenidos por el AG para el criterio de error ISE.

\begin{tabular}{|c|c|}
\hline Valor del error ISE & 169.52 \\
\hline$k_{t m d}$ & $16586.30 \mathrm{kgm}^{2} / \mathrm{s}^{2}$ \\
$d_{t m d}$ & $13692.26 \mathrm{kgm}^{2} / \mathrm{s}$ \\
$m_{t m d}$ & $38542.14 \mathrm{~kg}$ \\
\hline
\end{tabular}

En la Fig. 5 se representa el ángulo de desplazamiento de la torre: en azul el desplazamiento de la estructura sin TMD, en rojo con TMD con los parámetros 
iniciales, y en naranja con el TMD con los parámetros optimizados. Se puede ver a simple vista cómo se ha reducido el balanceo de la torre al incluir un sistema de control pasivo, que logra estabilizarse sobre los 600s (la simulación es de $1400 \mathrm{~s}$ ), y cómo con el control optimizado se mejora notablemente, consiguiendo eliminar las oscilaciones a los $200 \mathrm{~s}$. Además, la amplitud de las oscilaciones también se reduce desde el inicio.

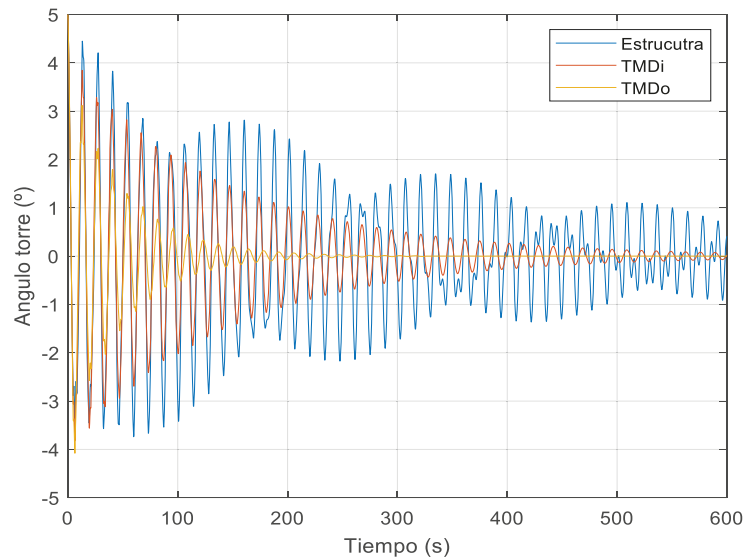

Figura 5: Angulo de desplazamiento de la torre sin TMD (azul), con TMD (roja) y con TMD optimizado con el criterio ISE (naranja).

Para realizar las comparaciones entre los distintos criterios de error que se proponen para la optimización se ha utilizado la desviación estándar de la torre, con y sin dispositivo TMD, definida en la ecuación (8). Esta ecuación representa el porcentaje de mejora en función de la oscilación que se produce en la torre con el dispositivo TMD respecto a la oscilación que se produce en la torre sin el mismo.

$\varepsilon=\frac{\sigma-\sigma_{T M D}}{\sigma} \cdot 100 \%$

En la Fig. 6 se muestra la respuesta de la señal en frecuencia obtenida mediante la transformada rápida de Fourier, para analizar cómo se distribuye la potencia que genera la estructura a la frecuencia donde se producen la mayor parte de vibraciones ( $\sin$ TMD en azul, con TMD línea roja y con TMD optimizado con el criterio ISE en naranja). Se puede observar que con el dispositivo TMD incorporado en la estructura no sólo disminuye la potencia disipada por las vibraciones, sino que además se elimina prácticamente el primer armónico generado en la estructura. Con el TMD optimizado con genéticos la reducción de la potencia es todavía mayor, como se comentará atendiendo a los resultados de la Tabla 7.

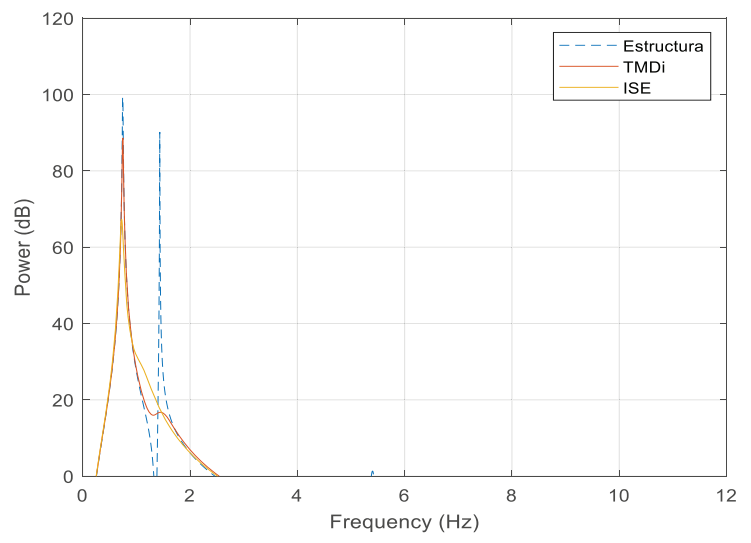

Figura 6: Respuesta en frecuencia de la estructura (azul), con TMD (rojo) y con TMD optimizado con criterio ISE (naranja).

Por último, se muestran en las tres gráficas de la Figura 7 el desplazamiento de la torre (primera figura), de la plataforma (gráfica del medio) y de la masa del TMD con el control pasivo sin optimizar y optimizado según este criterio de error ISE.
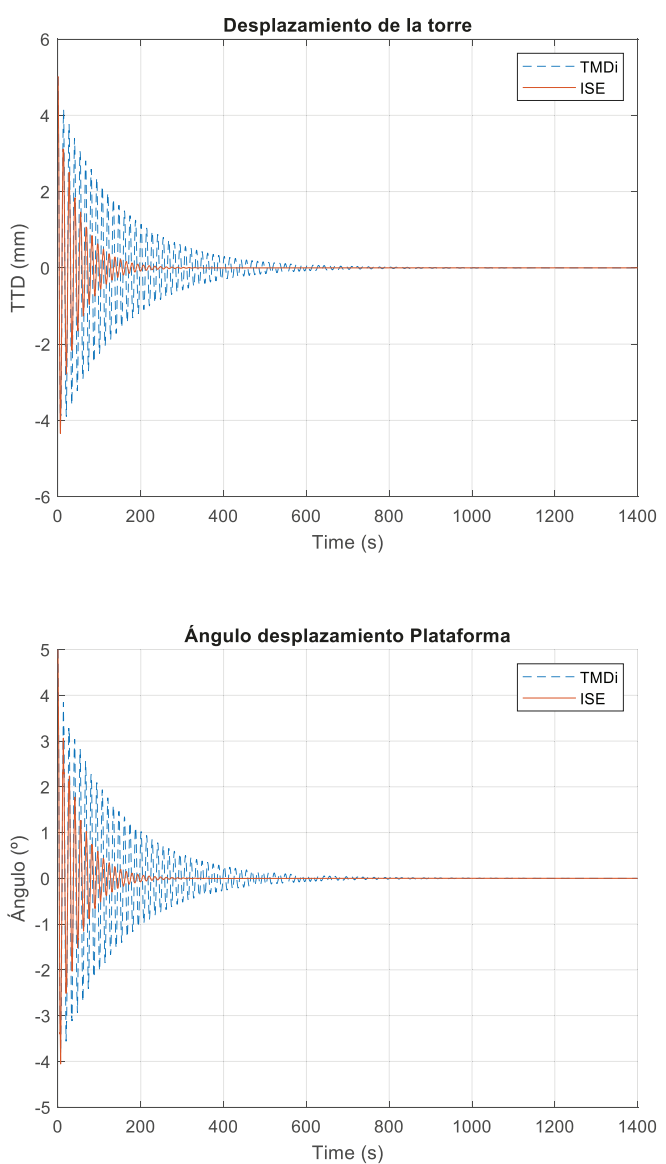


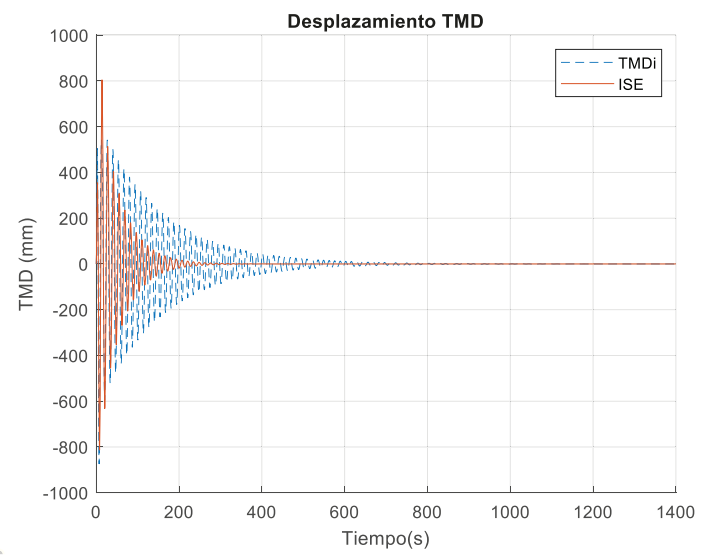

Figura 7: Desplazamiento de la torre (arriba), de la plataforma (en medio) y de la masa del TMD (abajo), con el control pasivo sin optimizar (azul) y optimizado (rojo), según el criterio de error ISE.

Se puede observar en todas ellas la reducción de la amplitud de las oscilaciones y la disminución del tiempo en el que el sistema se estabiliza y deja de oscilar.

\subsection{COMPARACIÓN DE RESULTADOS PARA LOS CUATRO CRITERIOS DE ERROR}

En la Tabla 6 se resumen los valores de los parámetros del TMD obtenidos con cada uno de los 4 criterios de error usados para la optimización. En la última fila se muestran los valores del TMD sin optimizar, con los parámetros de [8].

Tabla 6: Resultados de los parámetros obtenidos mediante la optimización del TMD con los cuatro criterios de error

\begin{tabular}{|c|c|c|c|}
\hline & $\begin{array}{c}\boldsymbol{k}_{\text {tmd }} \\
{\left[\mathbf{k g m}^{2} / \boldsymbol{s}\right]}\end{array}$ & $\begin{array}{c}\boldsymbol{d}_{\text {tmd }} \\
{\left[\mathbf{k g m}^{2} / \mathbf{s}^{2}\right]}\end{array}$ & $\begin{array}{c}\boldsymbol{m}_{\text {tmd }} \\
{[\mathbf{k g}]}\end{array}$ \\
\hline $\boldsymbol{I A E}$ & 15584.83 & 12689.25 & 39606.54 \\
\hline $\boldsymbol{I S E}$ & 15224.58 & 11677.91 & 39396.10 \\
\hline $\boldsymbol{I T A E}$ & 16303.59 & 14849.14 & 39867.02 \\
\hline $\boldsymbol{I T S E}$ & 15110.30 & 14978.49 & 39435.44 \\
\hline $\boldsymbol{T M D}$ & 28885.00 & 10183.00 & 40000.00 \\
\hline
\end{tabular}

Con esos valores de los elementos del TMD, en la Tabla 7 se muestran los resultados de simulación del sistema cuando se optimiza el TMD según los diversos criterios de error. En la primera fila se muestra el factor de mejora de la desviación de la torre dado por la ecuación (8). En las siguientes filas se ha calculado la potencia en la frecuencia de resonancia y en el primer armónico, junto a su valor en porcentaje respecto a la potencia generada en la estructura de la FOWT sin el TMD. La columna con etiqueta TMD muestra esos valores de la estructura cuando se ha usado el TMD sin optimizar, y la última columna, FOWT, la vibración de la turbina sin control pasivo.
Tabla 7: Análisis de las señales

\begin{tabular}{|c|c|c|c|c|c|c|}
\hline & IAE & ISE & ITAE & ITSE & TMD & FOWT \\
\hline $\boldsymbol{\varepsilon}(\%)$ & 58.76 & 59.23 & 57.59 & 59.25 & 30.02 & - \\
\hline Potencia $_{\boldsymbol{r}}(\mathrm{dB})$ & 67.56 & 67.24 & 68.13 & 65.96 & 88.59 & 99.24 \\
\hline Potencia $_{\boldsymbol{n}}(\mathrm{dB})$ & 17.04 & 17.06 & 16.72 & 16.68 & 16.75 & 90.18 \\
\hline Potencia $\boldsymbol{f}_{\boldsymbol{r}}(\%)$ & 32.03 & 32.24 & 31.34 & 33.53 & 81.50 & - \\
\hline Potencia $\boldsymbol{f}_{n}(\%)$ & 81.10 & 81.08 & 81.45 & 81.50 & 81.42 & - \\
\hline
\end{tabular}

Analizando el caso para el criterio de error ISE, hay una mejora en las oscilaciones de la torre respecto a la estructura sin TMD de un $59.23 \%$, factor que se puede apreciar en la Fig. 7, donde se estabiliza aproximadamente a los 200s. También esta mejora es mayor que si se aplica el TMD con los valores iniciales, sin optimizar. Analizando la respuesta en frecuencia del sistema en la Fig. 6 observamos en la frecuencia de resonancia una reducción de la potencia de un $32.24 \%$, mientras que en el primer armónico el factor de reducción llega a ser de un $81.08 \%$.

Con respecto a los demás criterios de error, como se comentó, los resultados son muy similares.

\section{CONCLUSIONES Y TRABAJO FUTURO}

En este trabajo se muestra la mejora de la respuesta de una FOWT respecto a reducción de las vibraciones al optimizar un dispositivo de control pasivo TMD mediante algoritmos genéticos. Se han probado cuatro criterios de error que tradicionalmente se usan en la evaluación de los sistemas de control.

Se ha comprobado que incluir en la estructura un dispositivo de control pasivo reduce la carga que sufre el sistema eólico marino, reduciendo los efectos de fatiga, lo que se pueden traducir en un aumento de la vida útil y una disminución del mantenimiento, factores que frenan el desarrollo de estos generadores de energía.

Como trabajo futuro se plantea integrar los métodos de optimización en el software FAST [2], una plataforma que simula las turbinas flotantes incluyendo factores hidrostáticos y aerodinámicos, obteniendo un sistema más cercano a la realidad donde estudiar los efectos de cada criterio de error en función de las condiciones externas. Además, se quiere seguir investigando en la posibilidad de aplicar optimización multicriterio.

\section{Agradecimientos}

Este trabajo ha sido parcialmente financiado por el Proyecto RTI2018-094902-B-C21, del Ministerio de Ciencia, Innovación y Universidades. 


\section{English summary}

\section{HEURISTIC OPTIMIZATION OF TMD CONTROL IN FLOATING WIND TURBINES}

\begin{abstract}
Among the current deployment of renewable energy systems, floating wind turbines are a promising resource. They take advantage of the wind at deep seas, where it reaches higher and more constant speed. However, being located in deep waters they are subject to heavy loads, caused mainly by waves and wind. To reduce the vibrations in the structure, passive control devices can be used, which come from civil engineering. But its design and tuning are not a simple task due to the diverse goals that coexist in the application of these passive control systems. In this work the use of different methods of signal analysis for the optimization of TMD devices (Tuned Mass Damper) is explored through genetic algorithms. Various error criteria have been applied for the optimization in order to obtain a greater reduction of vibrations in the various elements of the floating turbine.
\end{abstract}

Keywords: Modelling and simulation, Optimization, Floating Offshore Wind Turbines, Genetic Algorithms, Renewable energy.

\section{Referencias}

[1] Alonso-Zotes, F., Santos, M., (2017) Heuristic optimization of interplanetary trajectories in aerospace missions. Revista Iberoamericana de Automática e Informática Industrial 14(1), 1-15.

\section{[2] FAST (2019) https://nwtc.nrel.gov/FAST}

[3] Jonkman, J., Butterfield, S., Musial, W., and Scott, G., (2008) Definition of a 5-MW Reference Wind Turbine for Offshore System Development, TP 500-38060, National Renewable Energy Laboratory

[4] Pathak, N., Verma, A., \& Bhatti, T. S., (2016) New optimization criterion for load frequency control of power system. In 2016 IEEE 1st International Conference on Power Electronics, Intelligent Control and Energy Systems (ICPEICES) (pp. 1-5). IEEE.
[5] Santos, M., \& Cantos, A. J., (2010). Classification of plasma signals by genetic algorithms. Fusion Science and Technology, 58(2), 706-713.

[6] Santos, M. (2011). Un enfoque aplicado del control inteligente. Revista Iberoamericana de Automática e Informática Industrial RIAI, 8(4), 283-296.

[7] Stewart, G. M., Lackner, M. A., (2011) "The effect of actuator dynamics on active structural control of offshore wind turbines". Engineering Structures, 33(5), 1807-1816.

[8] Tomás-Rodríguez, M., Elsaghir, T., Hashi, S., Santos M., (2018) Análisis de vibraciones en turbinas marinas. Actas de las XXXIX Jornadas de Automática, 764-769.

[9] Tomás-Rodríguez, M., Santos, M., (2019) Modelling and control of floating offshore wind turbines. Revista Iberoamericana de Automática e Informática Industrial, in press.

[10] Tomas-Rodriguez, M., Feroz, K., Santos, M. (2019) Floating offshore wind turbines oscillations damping. 10th EUROSIM Congress, La Rioja, Logroño, Spain, July 1-5, 2019. ISBN 978-3-901608-92-6, DOI 10.11128/arep.58

[11] Villoslada, D., Santos M., Tomás-Rodríguez, M. (2019). Identification and validation of a barge floating offshore wind turbine model with optimized Tuned Mass Damper. 10th EUROSIM Congress, La Rioja, Logroño, Spain, July 1-5, 2019. ISBN 978-3-901608-92-6, DOI 10.11128/arep.58

[12] Yang, J., He, E. M., \& Hu, Y. Q., (2019). Dynamic modeling and vibration suppression for an offshore wind turbine with a tuned mass damper in floating platform. Applied Ocean Research, 83, 21-29.

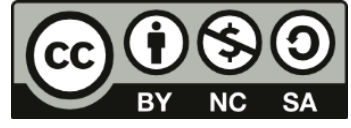

(C) 2019 by the authors. Submitted for possible open access publication under the terms and conditions of the Creative Commons Attribution CC BY-NC-SA 4.0 license (https://creativecommons.org/licenses/bync-sa/4.0/deed.es). 